\title{
ATUAÇÃO DA EQUIPE DE ENFERMAGEM FRENTE ACIDENTES COM MATERIAIS PERFUROCORTANTES EM UM HOSPITAL ESCOLA
}

\section{NURSING TEAM ACTING ACCIDENTS WITH PERFOROCATING MATERIALS IN A SCHOOL HOSPITAL}

\author{
Ana Carolina Ferro, Suellen Vienscoski Skupien ${ }^{1 *}$, Caroline Gonçalves Pustiglione \\ Campos $^{1}$, Elaine Cristina Antunes Rinaldi ${ }^{1}$ \\ ${ }^{1}$ Universidade Estadual de Ponta Grossa, Ponta Grossa, Paraná, Brasil \\ *Autor correspondente: Suellen Vienscoski Skupien Rua Luiz Nadal Motti, 319, CEP 84016- \\ 270 - Ponta Grossa, Paraná, Brasil E-mail: suvienscoski@hotmail.com Telefone: (42) \\ 999142927
}

\begin{abstract}
RESUMO
Os acidentes de trabalho apresentam alta incidência entre os profissionais de saúde devido aos inúmeros riscos ocupacionais a que esses trabalhadores estão expostos. Conforme dados do Ministério do Trabalho, no Brasil ocorreram 704.136 acidentes de trabalho no ano de 2014, desses 59.080 estão relacionados ao atendimento hospitalar. Objetivou-se caracterizar o acidente com material perfurocortante e retratar a forma de intervenção dos enfermeiros frente aos riscos ocupacionais em um hospital escola. Tratase de estudo exploratório e retrospectivo, realizado em junho de 2016. Os dados foram coletados por meio das fichas de Comunicado de Acidente de Trabalho e entrevista com enfermeiros. Os resultados apontam que os acidentes ocorreram prevalentemente com profissionais do sexo feminino, uma vez que a Enfermagem é majoritariamente composta por mulheres; como local, as mãos foram as regiões mais atingidas; o período de maior ocorrência dos acidentes de trabalho foi o matutino; falta de notificação de todos os acidente de trabalho que ocorrem na instituição, bem como o desconhecimento da Norma Regulamentadora 32 por parte dos profissionais entrevistados. Conclui-se que os profissionais de Enfermagem estão diariamente expostos a riscos ocupacionais, principalmente ao biológico por meio do trabalho e desenvolvimento de atividades assistenciais tornando-se primordial o conhecimento dos profissionais a respeito da Norma Regulamentadora 32, que pode ser adquirido e aprimorado por meio de educação continuada em serviço, com o objetivo de diminuir os riscos no ambiente de trabalho bem como garantir a segurança e a saúde do trabalhador no desenvolver da prática da assistência de Enfermagem.
\end{abstract}

Palavras-chave: Acidentes de trabalho; Riscos ocupacionais; Equipe de Enfermagem; Saúde do trabalhador.

\begin{abstract}
Occupational accidents have a high incidence among health professionals due to the numerous occupational risks to which these workers are exposed. According to data from the Ministry of Labor, in Brazil there were 704,136 occupational accidents in 2014 , of these 59,080 are related to hospital care.The objective of this study was to characterize the accident with sharps and to portray the nurses' intervention in face to occupational risks in a teaching hospital. This is an exploratory and retrospective study, conducted in June 2016. Data were collected through the forms of Work Accident Report and interview with nurses. The results indicate that the accidents occurred predominantly with female professionals, since nursing is mostly composed of women; as local, the hands were the hardest hit regions; the period of greatest occurrence of occupational accidents was the morning; lack of notification of all occupational accidents that occur in the institution, as well as ignorance of the Regulatory Standard 32 by the
\end{abstract}


interviewed professionals. It is concluded that Nursing professionals are daily exposed to occupational risks, especially to biological risks through work and development of care activities, making it essential for professionals to know about Regulatory Standard 32, which can be acquired and improved through continuing education in service, with the objective of reducing risks in the workplace as well as ensuring the safety and health of workers in the development of nursing care practice.

Keywords: Accidents occupational; Occupational risks; Nursing team; Occupational health.

\section{INTRODUÇÃO}

Os acidentes de trabalho apresentam alta incidência entre os profissionais de saúde devido aos inúmeros riscos ocupacionais a que esses trabalhadores estão expostos. Conforme dados do Anuário Estatístico da Previdência Social ${ }^{(1)}$, no Brasil ocorreram 704.136 acidentes de trabalho no ano de 2014, desses 59.080 correspondem a atividades relacionadas ao atendimento hospitalar.

No ambiente hospitalar, a atividade da equipe de Enfermagem presta assistência durante período integral ao paciente, dessa forma pode-se afirmar que são os profissionais mais expostos a riscos de acidentes, dentre eles, os acidentes com materiais perfurocortantes e com isso a chance de contaminação com materiais biológicos ${ }^{(2)}$.

O risco biológico é caracterizado pela probabilidade de contaminação por materiais biológicos advindos de fluidos corpóreos do paciente que podem conter vírus, bactérias e parasitas, onde em um acidente pode entrar em contato com a pele, mucosas ou sangue do profissional de saúde. Este risco está intimamente ligado aos acidentes com perfurocortantes, pois os objetos que os causam (como agulhas, cateteres venosos, pinças, tesouras, entre outros) podem conter restos de materiais biológicos dos pacientes em que foram usados ${ }^{(2,3)}$.

No Brasil, a preocupação com a contaminação por fluidos biológicos surgiu na década de 80 , com a epidemia da Síndrome da Imunodeficiência Adquirida, quando começaram a se adotar medidas preventivas para com esses acidentes ${ }^{(3)}$.

Nesta perspectiva, implantou-se a Norma Regulamentadora 32 (NR 32), a qual estabelece diretrizes básicas para proteger a segurança e a saúde dos trabalhadores da área da saúde, com o objetivo de prevenir acidentes e o adoecimento causados pelo trabalho nos profissionais, recomendando para cada situação de risco, medidas preventivas. Dentre as disposições estabelecidas pela NR 32 está o uso de vestimentas adequadas para o ambiente de trabalho; a vacinação contra hepatite $\mathrm{B}$, tétano e difteria; cuidados com os alimentos; a higienização das mãos; entre outras disposições ${ }^{(4)}$.

A NR 32 ainda aponta sobre a importância do empregador fornecer aos seus empregados capacitações sobre a correta utilização dos dispositivos de segurança, além da elaboração e implantação do Plano de Prevenção de Riscos de Acidentes com Materiais Perfurocortantes, traçando-se um plano de prevenção a acidentes que possam ocasionar exposição a agentes biológicos $^{(4)}$.

Outra medida preventiva implantada pelo Ministério do Trabalho em 2012 foi o Plano Nacional de Segurança e Saúde no Trabalho, o qual tem por objetivos a promoção da saúde e a melhoria da qualidade de vida do trabalhador, assim como a prevenção de acidentes e danos à saúde, que se relacionam ao trabalho ou que ocorram na execução dele, por meio da eliminação e redução dos riscos nos ambientes de trabalho ${ }^{(5)}$.

Apesar de todas as medidas preventivas, os acidentes com materiais perfurocortantes são frequentes entre os profissionais de Enfermagem, porém, não há um número real desses acidentes devido à subnotificação. Acredita-se que a subnotificação seja decorrente da falta de conscientização dos profissionais de saúde ou mesmo pelo medo e culpa que sentem em relação ao acidente $^{(2)}$.

Este estudo justifica-se pela necessidade de sensibilizar a equipe de Enfermagem sobre os riscos ocupacionais e também de incentivar a notificação em casos de ocorrência do acidente, já que a subnotificação destes, pelos profissionais de Enfermagem, impossibilita a instituição de conhecer sobre a grandeza do 
problema, inviabilizando o planejamento e o desenvolvimento de ações preventivas e de controle.

Para que o enfermeiro possa perceber os riscos e solucioná-los, é importante que seja conhecedor da sua equipe, visto que apenas a aplicação de precauções e intervenções no processo de trabalho não são suficientes para garantir as medidas de prevenção, devendo fazer parte das estratégias as reflexões a respeito das mudanças de comportamento e as causas dos acidentes e favorecendo a prevenção dos acidentes de trabalho ${ }^{(6)}$.

Portanto, este estudo objetiva caracterizar o acidente com material perfurocortante e retratar a forma de intervenção dos enfermeiros frente aos riscos ocupacionais em um hospital escola no Paraná.

\section{METODOLOGIA}

Esta pesquisa tem abordagem quanti-qualitativa do tipo exploratória, retrospectiva, realizada em um hospital escola no município de Ponta Grossa, Paraná, dividida em duas etapas: (etapa 1) análise documental das fichas de Comunicado de Acidente de Trabalho (CAT) e (etapa 2) entrevista com enfermeiros.

Para a etapa 1 tem-se como sujeitos de pesquisa os profissionais de Enfermagem que se acidentaram com materiais perfurocortantes no período de janeiro de 2011 a dezembro de 2015, os quais foram notificados e arquivados na Comissão de Controle de Infecção Hospitalar e Serviço Especializado em Segurança e Medicina do Trabalho. A coleta de dados consistiu na consulta às fichas de CAT, disponibilizadas pelo setor de Recursos Humanos, constando a relação de profissionais que se acidentaram de alguma forma na referida instituição, dentre estas os acidentes com materiais perfurocortantes.

Os dados coletados na etapa 1 foram digitados em planilhas do programa Excel e analisados por meio de estatística descritiva, utilizando-se o software Statistical Package for Social Sciences (SPSS), versão 17.

Para a etapa 2, realizaram-se entrevistas semiestruturadas com enfermeiros, em seus gabinetes, no período de junho de 2016, com áudio gravadas e posteriormente transcritas. As entrevistas tiveram uma duração média de 30 minutos. Utilizou-se um roteiro contendo as seguintes questões: Qual o papel do enfermeiro quando o assunto é saúde do trabalhador?; O que você conhece sobre a Norma Regulamentadora 32?; Conhece a Comissão Interna de Prevenção de
Acidentes da instituição em que trabalha?; O que você faz para conservar a saúde dos seus funcionários?.

Ainda referente à etapa 2, o convite para as entrevistas foi realizado via telefone a 06 enfermeiros, atuantes na Unidade de Terapia Intensiva Neonatal, Unidade de Terapia Intensiva Adulto, Centro Cirúrgico, Clínica Cirúrgica, Clínica Médica e Pronto Atendimento. A escolha justifica-se por estes setores por serem considerados semicríticos e críticos dentro da referida instituição. Para a garantia do anonimato dos participantes, estes foram denominados com a letra "E" seguida do número da entrevista como E1, E2, E3, E4, E5 e E6. Ressalta-se que todos os entrevistados assinaram o Termo de Consentimento Livre e Esclarecido.

Os dados coletados na etapa 2 foram analisados por meio da análise de conteúdo de Bardin ${ }^{(7)}$, organizada em três fases: pré-análise; exploração do material e tratamento dos resultados; e interpretação.

Após a organização dos dados, estes foram expressos em quatro categorias a saber: Categoria $1-\mathrm{O}$ papel do enfermeiro na saúde do trabalhador; Categoria 2 - Conhecimento dos enfermeiros sobre a Norma Regulamentadora 32; Categoria 3 - Comissão Interna de Prevenção de Acidentes e equipe de enfermagem; e Categoria 4 - Educação continuada e qualidade de vida.

Este estudo atendeu aos princípios éticos, sendo aprovado pelo Comitê de Ética e Pesquisa com Seres Humanos da Universidade Estadual de Ponta Grossa, sob parecer $n^{\circ} 1.520 .222$.

\section{RESULTADOS}

No total, 20 profissionais de Enfermagem se acidentaram de alguma forma com material perfurocortante. Destes, 17 (85\%) pertencem ao sexo feminino e $03(15 \%)$ ao sexo masculino, sendo que a parte do corpo mais atingida foram às mãos com 18 (90\%) acidentes.

Quanto ao turno de trabalho, os acidentes ocorreram em sua maioria no período matutino, onde 12 (60\%) profissionais de Enfermagem se acidentaram. O setor em que os profissionais da Enfermagem exercem suas atividades laborais com maior índice de acidentes foi a clínica cirúrgica com 06 (30\%). Cabe salientar que todos os dados descritos acima estão na Tabela 1 . 
Tabela 1 - Perfil dos profissionais de enfermagem quanto ao acidente por perfurocortante. Ponta Grossa, PR, Brasil, 2011-2015.

\begin{tabular}{|c|c|}
\hline Variável & N (\%) \\
\hline \multicolumn{2}{|l|}{ Sexo } \\
\hline Feminino & $17(85)$ \\
\hline Masculino & $3(15)$ \\
\hline \multicolumn{2}{|l|}{ Idade } \\
\hline $22-34$ & $13(65)$ \\
\hline $35-51$ & $7(35)$ \\
\hline \multicolumn{2}{|l|}{ Ocupação } \\
\hline Técnico de enfermagem & $14(70)$ \\
\hline Enfermeiro & $6(30)$ \\
\hline \multicolumn{2}{|l|}{$\begin{array}{l}\text { Local do corpo atingido por } \\
\text { material perfurocortante }\end{array}$} \\
\hline Mãos & $18(90)$ \\
\hline Cervical & $1(5)$ \\
\hline Braço & $1(5)$ \\
\hline \multicolumn{2}{|l|}{ Turno de trabalho } \\
\hline Matutino & $12(60)$ \\
\hline Vespertino & $6(30)$ \\
\hline Noturno & $2(10)$ \\
\hline \multicolumn{2}{|l|}{ Setor de trabalho } \\
\hline Clínica médica & $4(20)$ \\
\hline Clínica cirúrgica & $6(30)$ \\
\hline Central de materiais & $2(10)$ \\
\hline Centro cirúrgico & $2(10)$ \\
\hline Pronto atendimento & $2(10)$ \\
\hline Unidade de terapia intensiva & $4(20)$ \\
\hline
\end{tabular}

Fonte: dados da pesquisa.

Os resultados obtidos na etapa 2 serão descritos a seguir de acordo com as categorias.

\section{Categoria 1 - O papel do enfermeiro na saúde do trabalhador}

Pode-se ver nos depoimentos a importância do enfermeiro frente à equipe técnica:

$E$ E o enfermeiro que vai tomar frente do caso de um acidente, preenchendo a CAT, encaminhando esse funcionário a consulta no próprio Pronto Atendimento do hospital, porém sabemos que muitos colegas não sabem como proceder quando acontece um acidente com material biológico. (E1)

[...] fazer toda a parte de gestão da parte de pessoas, também a questão de educação em saúde, a própria prevenção de acidentes, e quando há acidentes saber direcionar o funcionário sobre como proceder frente a isso. (E4)
Outro entrevistado reforça:

[...] o enfermeiro deve sempre pensar na equipe em que esta gerenciando, pois não é apenas o contato com os materiais biológicos em um eventual acidente que preocupa, mas também a saúde mental desse funcionário que se altera. (E6)

\section{Categoria 2 - Conhecimento dos enfermeiros sobre a Norma Regulamentadora 32}

Sobre a NR 32, a qual tem por finalidade estabelecer as diretrizes básicas para a implementação de medidas de segurança e proteção aos que trabalham em serviços de saúde, e aqueles que exercem atividades de promoção e assistência a saúde geral, 4 enfermeiros mostraram dificuldades em responder a questão e 2 relataram não saber do que se tratava.

A NR 32 é uma norma regulamentadora em serviços [...], ela fala bastante sobre a questão do cuidado pessoal, a utilização de calçados fechados, avental, unhas curtas, brincos. (E2)

[...] a utilização de EPI's, também se não me engano do descarte correto dos resíduos gerados pela saúde. (E4)

[...] norma que regulamenta os cuidados que os profissionais da área da saúde têm de como está a vestimenta, os cuidados assim para a segurança do trabalhador. Ela trata de riscos biológicos e químicos e também o descarte de resíduos. (E5)

Neste contexto o diálogo assume outra conformação:

Eu lembro muito pouco, pois são tantas normas que eu não sei muito bem. Ela é aquela normativa que regulamenta o uso do EPI dentro da instituição. (E2)

[...] a eu sei que a NR 32 regulamenta a atividade no hospital, o hospital como um todo, fala dos direitos e deveres da equipe de saúde, fala sobre os EPI's, a própria estrutura do hospital, eu já li mais eu não lembro muito bem assim. (E3)

\section{Categoria 3 - Comissão Interna de Prevenção de Acidentes e equipe de enfermagem}

Os enfermeiros identificaram dificuldades sobre a atuação da CIPA do hospital, necessitando de melhorias na sua efetivação.

A CIPA é norteada pela Norma Regulamentadora 5 , que objetiva à prevenção de acidentes e doenças decorrentes do trabalho, de forma que torna compatível o trabalho com a preservação da vida e a promoção da saúde do trabalhador. 
Participo da CIPA, mas acredito que falta muito para funcionar dentro da instituição, trabalhamos com a Comissão, de maneira mais assim de levantar dificuldades, como a questão do estrutural e a Semana Interna de Prevenção de Acidentes de Trabalho. (E4)

[...] conheço a CIPA, na verdade eu sei que tem, porém se for pensar em efetividade em relação ao serviço prestado aos funcionários dentro da instituição, não tem efetividade nenhuma [...], pois o serviço ainda visa apenas o risco do paciente. (E2)

[...] participei da segunda CIPA da instituição, porém se me perguntar se é efetiva com o trabalhador acredito que poderia ser melhor [...], talvez mais contato direto com o funcionário, saber quais são os seus problemas, questionamentos [...], a CIPA para eu, se mostra mais voltada a uma grande preocupação com a segurança do paciente. (E5)

Outro entrevistado reforça:

[...] pois só ouvimos falar da Cipa no curso anual, em outras atividades assim pode ser por ignorância minha, porém nunca ouvi falar. (E3)

\section{Categoria 4 - Educação continuada e qualidade de vida}

[...] para conservar a saúde do meu funcionário, eu procuro fazer capacitação e orientar, mas cada vez que a gente vê um perigo em potencial, faz a capacitação novamente. (E1)

[...] uma coisa que eu institui dentro do setor é que usem máscara para preparar a medicação, não é questão de contaminar a medicação sabe, mais sim do funcionário estar se contaminando, estar inalando na hora de preparar a diluição [...], e isso eu vi que era um risco eminente para a saúde deles. (E2)

Eu trabalho muito com educação continuada, com descarte adequado de resíduos, sempre que tem algo descartado incorretamente eu procuro chamar o pessoal e orientar [...], além disso eu trabalho bastante com precaução padrão e precaução de contato, o uso de EPI's em si, pelos isolamentos que possuímos no setor. (E4)

Além disso, os entrevistados também se mostram preocupados com a questão psicológica e mental dos profissionais:

Além da orientação e capacitação, dos usos dos EPI's, também é importante o cuidado mental, para que assim se consiga desenvolver um bom trabalho. (E6)

[...] tento sempre estar conversando com eles, fazendo capacitação, e também cuidar da parte psicológica dos funcionários, a gente agora está conversando junto com a psicóloga do hospital e também junto com os acadêmicos de psicologia, eles estão vindo ao hospital pra atender os funcionários, a gente tem feito então um trabalho nesse sentido, principalmente para ofertar um trabalho psicológico para os funcionários que estão mais abalados. (E5)

\section{DISCUSSÃO}

Esta pesquisa mostra a prevalência do sexo feminino na área da Enfermagem na referida instituição. Esses resultados convergem com outros estudos, uma vez que a equipe de Enfermagem é majoritariamente composta por mulheres, as quais, talvez, estejam mais aptas a desempenhar o cuidado direto e contínuo ao paciente $^{(8,9)}$.

Além do sexo feminino, neste estudo há prevalência de acidentes com técnicos de enfermagem. Segundo estudos ${ }^{(10,11)}$, esses dados podem estar ligados as atividades que cada categoria exerce dentro do hospital, pois a equipe técnica é quem está à frente dos procedimentos, tendo maior contato com o paciente, dessa forma estando mais vulneráveis a questão de acidentes pelas atividades desenvolvidas pelos mesmos, como o manuseio e preparo de materiais para os procedimentos ou mesmo a organização do âmbito de trabalho.

Neste estudo, observou-se que a maior ocorrência dos acidentes com perfurocortante se deu no período diurno, especialmente no matutino. Através de estudo, justifica-se que é o momento, com maior intensidade de trabalho assistencial, dessa forma, o ritmo de trabalho no período matutino é mais intenso principalmente pela equipe técnica, a qual realiza seus procedimentos como medicação, diluição de medicamentos associados a procedimentos como banho de leitos, o que pode vir a tomar algum tempo em que pode estar acelerando os procedimentos que envolvam perfurocortantes, ocasionando muitas vezes acidentes ${ }^{(11)}$.

Neste estudo, a parte do corpo mais atingida nos acidentes de trabalho com perfurocortante foram as mãos. Segundo estudo ${ }^{(12)}$ as mãos geralmente são as partes mais atingidas pelos acidentes com perfuro cortantes, pois geralmente está em contato com o paciente, na administração de medicamentos, ou mesmo qualquer procedimento que venha a utilizar algum tipo de agulha ou instrumental que possa estar causando algum tipo de lesão.

Outro estudo aponta que no Hospital de Montes Claros, a parte do corpo mais atingida pelos acidentes também foram as mãos. Justifica-se pelo fato de que 
a Enfermagem é um trabalho predominantemente manual onde exigem destreza e precisão ${ }^{(13)}$.

Pode-se perceber que neste estudo, grande parte dos acidentes ocorreu no setor da clínica cirúrgica. Esse resultado vem ao encontro de estudos ${ }^{(14,15)}$ que mostraram que a clínica cirúrgica, médica e unidade de terapia intensiva estão ligados a altos índices de acidentes pelo grande fluxo de pacientes e pela assistência diferenciada aos pacientes, sendo pela necessidade de agilidade nos procedimentos, ou mesmo pela sobrecarga de trabalho que esses profissionais estão expostos ${ }^{(16)}$.

Para coordenar uma equipe, é preciso que o enfermeiro tenha conhecimento e sensibilidade para captar as necessidades dos seus funcionários, assim estimulando-os a ações inovadoras para que se adaptem a diferentes situações, onde assim poderão estar evitando um ocasional acidente de trabalho, organizando o seu cuidado e desenvolvendo um trabalho dinâmico e longe de $\operatorname{riscos}^{(17)}$.

Os profissionais enfermeiros que estão à frente de uma equipe, têm a necessidade de estar atentos as consequências negativas causadas pelos acidentes, que venham a afetar diretamente a vida dos trabalhadores. Estudos $^{(17,18)}$ relatam que todo acidente de trabalho envolvendo agentes e riscos biológicos necessitam da emissão da CAT, porém, muitas vezes, os acidentes são subnotificados pelos empregadores e também pelos empregados, que por sua vez perdem o amparo legal, e adquire o risco de estar contraindo uma doença que venha a acarretar prejuízos funcionais a sua vida.

Em relação à NR 32, foi possível observar que esta é conhecida superficialmente pelos enfermeiros. Segundo estudo ${ }^{(18)}$, a NR 32 aborda alguns pontos básicos à implementação de medidas de proteção e segurança, a quem trabalha nos serviços prestadores de saúde, dessa forma, se vê como fundamental o conhecimento dessa normativa, a fim de cumprir os deveres, reafirmar os direitos e principalmente promover a segurança no trabalho, prevenindo assim acidentes e doenças ocupacionais.

Outro estudo ${ }^{(19)}$ realizado com profissionais de outras instituições, como do Hospital de Valparaiso de Goiás, também conhecem superficialmente a NR 32, onde demonstram que sabem muito pouco, afirmando que a NR 32 vem a falar do uso de equipamentos de proteção individual nas atividades laborais.

Dessa forma, é necessário que haja um instrumento para tratar a prevenção de acidentes de trabalho e todas as condições e aspectos que afetam diretamente a saúde e a segurança do trabalhador. A CIPA tem como principal dever assegurar e promover boas condições de trabalho, minimizando os riscos de acidentes dentro das instituições ${ }^{(20)}$.

Estudos mostram que a CIPA não apenas na instituição estudada, mas também em outras se mostra ineficiente, sendo seu papel conscientizar os trabalhadores para que reconheçam riscos, proporcionar espaços para que os trabalhadores possam negociar mudanças e notificar situações, dessa forma tornando mais saudáveis e seguras as condições de trabalho ${ }^{(20)}$.

A atuação do enfermeiro vai muito além do saber tradicional em que volta sua atenção as práticas de um fazer técnico, o que se volta à saúde do paciente, mas, essa atenção volta-se, também, a construção coletiva do cuidado interagindo amplamente com todos os profissionais, coordenando não só o processo de saúde mais também os diferentes problemas e serviços do âmbito hospitalar ${ }^{(16)}$.

Apontou-se, ainda, a conservação da saúde dos funcionários, a qual se dá de forma a cada um preservar a sua saúde através do conhecimento adquirido pela orientação e pela educação em saúde, onde cada funcionário está apto a cuidar de sua própria saúde no âmbito de trabalho ${ }^{(16)}$.

Dessa forma, a atuação do enfermeiro sob o olhar da equipe e na organização hospitalar, desempenha importante papel nas relações da equipe de saúde, sendo referência pela sua liderança e valorização dos seus saberes.

O estudo mostra que a influência considerável por parte dos enfermeiros que aplicam educação continuada a sua equipe, além de complementar a formação do funcionário, também ajuda a estimular as habilidades, fazendo com que este acabe não só prestando uma assistência de qualidade ao paciente, mais também tendo a sua saúde preservada, evitando riscos que possam acarretar doenças permanentes ${ }^{(21)}$.

\section{CONCLUSÃO}

A equipe de Enfermagem está exposta diariamente a riscos biológicos, químicos e ergonômicos, dessa forma é de suma importância o conhecimento íntegro da NR 32 que vem a dar subsídios necessários para a garantia do trabalho seguro dentro da instituição, o que deixa a desejar por parte dos enfermeiros. 
O reduzido índice de acidentes com perfurocortantes na referida instituição, se comparada ao período estudado, pode ser justificado pela subnotificação, onde evidências apontam desinformação em relação aos riscos e aos aspectos epidemiológicos e jurídicos que envolvem este tipo de acidente em ambiente hospitalar.

Este estudo possui limitação, por não ter acesso na integra às fichas de CAT dentro da referida instituição, ocasionando a perda de informações consideradas relevantes.

Pode-se concluir, também, que cabe ao enfermeiro detectar os riscos eminentes no âmbito de trabalho, tomando decisões preventivas a partir da identificação desses riscos a que os profissionais de saúde estão expostos, mostrando a preocupação em torno da prevenção da saúde do trabalhador.

Dessa forma, salienta-se que o conhecimento transmitido por meio da educação continuada em saúde, dá continuidade ou mesmo início a um programa de prevenção e conscientização sobre os riscos a que esses profissionais estão diariamente expostos.

\section{REFERÊNCIAS}

1. Brasil. Ministério da Previdência Social. Anuário Estatístico da Previdência Social: AEPS-2014. Brasília: MTPS/DATAPREV, 2014.

2. Junior EPS, Batista RRAM, Almeida ATF, Abreu RAA. Acidente de trabalho com material perfurocortante envolvendo profissionais e estudantes da área da saúde em hospital de referência. Rev Bras Med Trab. 2015;13(2):69-75.

3. Espindola MCG, Fontana RT. Riscos ocupacionais e mecanismos de autocuidado do trabalhador de um centro de material e esterilização. Rev Gaúcha Enferm. 2012;33(1):116-23.

4. Brasil. Ministério do Trabalho e Emprego. Portaria n. 1.748, de 30 de agosto de 2011. Norma Regulamentadora 32. Dispõe sobre a Segurança e Saúde no Trabalho em Serviços de Saúde. Diário Oficial da União, 31 out 2011; Seção 1.

5. Brasil. Ministério da Previdência Social. Plano Nacional de Segurança e Saúde no Trabalho - Plansat. Brasília: CT-SST, 2012.

6. Santos PHS, Reis LA. Subnotificação de acidentes de trabalho em profissionais de enfermagem: revisão integrativa. Rev enferm UFPE on line. 2016;10(2):6406.
7. Bardin L. Análise de conteúdo. São Paulo: Edições 70; 2011.

8. Mota AGS. Siqueira JF. Pereira FGF. Freitas MMC. Rodrigues JLN. Caetano JA. Trabalhadores de um hospital escola em Fortaleza-CE: exposição cotidiana à Hepatite B. Vigilância sanitária em Debate, 2015;3(2):42-47.

9. Souza LL, Peres WS, Araújo DB. Problematizações de gêneros no campo da enfermagem: diálogos com feminismos e a teoria Queer. Revista NUPEM. 2015;7(13):121-42.

10. Oliveira QB, Santos RS, Santos CMF. Acidentes de trabalho na equipe de enfermagem: uma revisão de literatura. Rev Enfermagem Contemporânea. 2013;2(1):32-52.

11. Nowak NL, Campos GA, Borba EO, Ulbricht L, Neves EB. Fatores de risco para acidentes com materiais perfurocortantes. O Mundo da Saúde. 2013;37(4):41926.

12. Ottobelli C, Vaz MRC, Cargnin MCS, Argenta C, Zanatta RG. Acidentes de trabalho com perfurocortantes em unidade de centro cirúrgico na região sul do Brasil. O Mundo da Saúde. 2015;39(1):113-8.

13. Ruas EFG, Santos LS, Barbosa DA, Belasco AGS, Bettencourt ARC. Acidentes ocupacionais com materiais perfurocortantes em hospitais de Montes Claros-MG. Rev Min Enferm. 2012;16(3):437-43.

14. Januário GC. Carvalho PCF. Lemos GC. Gir E. Toffano S.E.M. Acidentes ocupacionais com material potencialmente contaminado envolvendo trabalhadores de enfermagem. Cogitare enferm. 2017; 22(1):01-9.

15. Rodrigues VS. Acidentes de trabalho da enfermagem com perfurocortantes em um Hospital Universitário: estratégias para prevenção. Uberlândia. Dissertação [Programa de Pós-graduação em Saúde Ambiental e Saúde do Trabalhador] - Instituto de Geografia da Universidade Federal de Uberlândia; 2017.

16. Ribeiro AS, Gabatz RIB, Neves ET, Padoin SMM. Caracterização de acidente com material perfurocortante e a percepção da equipe de enfermagem. Cogitare enferm. 2009; 14(4):660-6.

17. Silva CDL, Pinto WM. Riscos ocupacionais no ambiente hospitalar: fatores que favorecem a sua ocorrência na equipe de enfermagem. Saúde Colet Debate. 2012;2(1):95-105.

18. Pereira DMM. Costa NRC. Andrade MN. Torres DC. Rocha RSB. Avila PES. Conhecimento e adesão às 
práticas de biossegurança em um hospital de referência materno infantil. Para Res Med J. 2017;1(3):e23.

19. Rondon EC, Tavares MS, Santos WL. Fatores dificultadores e facilitadores que os profissionais de enfermagem enfrentam relacionados ao uso dos EPI'S. Revista Eletrônica Gestão e Saúde. 2012;3(3):676-82.

20. Aires RFF, Salgado CR, Neto LCS. Segurança e saúde no trabalho: estudo do funcionamento da CIPA de um hospital universitário. Tekhne e Logos. 2013; 4(2):1-13.

21. Bezerra ALQ, Queiroz ES, Weber J, Munari DB. O processo de educação continuada na visão de enfermeiros em um hospital universitário. Rev Eletr Enf. 2012;14(3):618-25. 